\title{
Filtered Ring Derived from Discrete Valuation Ring and Its Properties
}

\author{
M. H. Anjom Shoa*, M. H. Hosseini \\ University of Birjand, Birjand, Iran \\ Email: ${ }^{*}$ ajomshoamh@birjand.ac.ir, mhhosseini@birjand.ac.ir
}

Received 7 January 2014; revised 7 February 2014; accepted 15 February 2014

Copyright (C 2014 by authors and Scientific Research Publishing Inc.

This work is licensed under the Creative Commons Attribution International License (CC BY).

http://creativecommons.org/licenses/by/4.0/

(c) (i) Open Access

\section{Abstract \\ In this paper we show that if $R$ is a discrete valuation ring, then $R$ is a filtered ring. We prove some properties and relation when $R$ is a discrete valuation ring.}

\section{Keywords}

\section{Commutative Ring; Valuation Ring; Discrete Valuation Ring; Filtered Ring; Graded Ring; Filtered Module; Graded Module}

\section{Introduction}

In commutative algebra, valuation ring and filtered ring are two most important structures (see [1]-[3]). If $R$ is a discrete valuation ring, then $R$ has many properties that have many usages for example decidability of the theory of modules over commutative valuation domains (see [1]-[3]), Rees valuations, and asymptotic primes of rational powers in Noetherian rings, and lattices (see [4]). We know that filtered ring is also a most important structure since filtered ring is a base for graded ring especially associated graded ring, completion, and some results like on the Andreadakis Johnson filtration of the automorphism group of a free group (see [5]) on the depth of the associated graded ring of a filtration (see [6]). So, as important structures, the relation between these structures is useful for finding some new structure. In this article, we show that we can make a filtration with a valuation. Then we explain some new properties for it. On the other hand, we show this is a strongly filtered ring, then we explain some new properties for it.

\section{Preliminaries}

In this paper the ring $R$ means a commutative ring with unit.

"Corresponding author. 
Definition 2.1 A subring $R$ of a field $K$ is called a valuation ring of $K$, if for every $\alpha \in K, \alpha \neq 0$, either $\alpha \in R$ or $\alpha^{-1} \in R$.

Definition 2.2 Let $\Delta$ be a totally ordered abelian group. A valuation $v$ on $R$ with values in $\Delta$ is a mapping $v: R^{*} \rightarrow \Delta$ satisfying:

i) $v(a b)=v(a)+v(b)$;

ii) $v(a+b) \geq \min \{v(a), v(b)\}$.

Definition 2.3 Let $K$ be field. A discrete valuation on $K$ is a valuation $v: K^{*} \rightarrow Z$ which is surjective.

Definition 2.4 A fractionary ideal of $R$ is an $R$-submodule $M$ of $K$ such that $a M \subseteq R$, for some $a \in R, a \neq 0$.

Definition 2.5 A fractionary ideal $M$ is called invertible, if there exists another fractionary ideal $N$ such that $M N=R$.

Proposition 2.1 Let $R$ be a local domain. Every non zero fractionary ideal of $R$ is invertible if and only if $R$ is DVR (see [3]).

Theorem 2.1 Let $R$ be a Noetherian local domain with unique maximal ideal $m \neq 0$ and $K$ the quotient field of $R$. The following conditions are equivalent.

i) $R$ is a discrete valuation ring;

ii) $R$ is a principal ideal domain;

iii) $m$ is principal;

iv) $R$ is internally closed and every non-zero prime ideal of $R$ is maximal;

v) Every non-zero ideal of $R$ is power of $m$ (see [3]).

Definition 2.6 Let $R$ be a ring together with a family $\left\{R_{n}\right\}_{n \geq 0}$ of subgroups of $R$ if satisfying the following conditions:

i) $R_{0}=R$;

ii) $R_{n+1} \subseteq R_{n}$ for all $n \geq 0$;

iii) $R_{n} R_{m} \subseteq R_{n+m}$ for all $n, m \geq 0$;

Then we say $R$ has a filtration.

Definition 2.7 Let $R$ be a ring together with a family $\left\{R_{n}\right\}_{n \geq 0}$ of subgroups of $R$ if satisfying the following conditions:

i) $R_{0}=R$;

ii) $R_{n+1} \subseteq R_{n}$ for all $n \geq 0$;

iii) $R_{n} R_{m}=R_{n+m}$ for all $n, m \geq 0$;

Then we say $R$ has a strong filtration.

Example 2.1 Let $I$ be an ideal of $R$, then $R_{i}=I^{i}$ is a filtration that is called $I$ adic filtration ring.

Definition 2.8 Let $R$ be a filtered ring. A filtered $R$-module $M$ is an $R$-module together with family $\left\{M_{n}\right\}_{n \geq 0}$ of subgroup $M$ of satisfying:

1. $M_{0}=M$;

2. $M_{n+1} \subseteq M_{n}$ for all $n \geq 0$;

3. $R_{n} M_{m} \subseteq M_{n+m}$ for all $n, m \geq 0$.

Then we say $M$ has a filtration.

Definition 2.9 A map $f: M \rightarrow N$ is called a homomorphism of filtered modules, if: i) $f$ is $R$-module an homomorphism and ii) $f\left(M_{n}\right) \subseteq N_{n}$ for all $n \geq 0$.

Definition 2.10 A graded ring $R$ is a ring, which can expressed as a direct sum of subgroup $\left\{R_{n}\right\}_{n \geq 0}$ i.e. $R=\oplus_{n \geq 0} R_{n}$ such that $R_{n} R_{m} \subseteq R_{n+m}$ for all $n, m \geq 0$

Definition 2.11 Let $R$ be a graded ring. An $R$-module $M$ is called a graded $R$-module, if $M$ can be expressed as a direct sum of subgroups $\left\{M_{n}\right\}_{n \geq 0}$ i.e. $M=\oplus_{n \geq 0} M_{n}$ such that $R_{n} M_{m} \subseteq M_{n+m}$ for all $n, m \geq 0$.

Definition 2.12 Let $M$ and $N$ be graded modules over a graded ring $R$. A map $f: M \rightarrow N$ is called 
homomorphism of graded modules if: i) $f$ is $R$-module an homomorphism and ii) $f\left(M_{n}\right) \subseteq N_{n}$ for all $n \geq 0$.

Definition 2.13 Let $R$ be a filtered ring with filtration $\left\{R_{n}\right\}_{n \geq 0}$. Let $g r_{n}(R)=R_{n} / R_{n+1}$, and $\operatorname{gr}(R)=\oplus_{n \geq 0} g r_{n}(R)$. Then $\operatorname{gr}(R)$ has a natural multiplication induced from $R$ given

$$
\left(a+R_{n+1}\right)\left(b+R_{m+1}\right)=a b+R_{m+n+1}
$$

where $a \in R_{n}, b \in R_{m}$. This makes $R$ in to a graded ring. This ring is called the associated graded ring of $R$.

Definition 2.14 Let $M$ be a filtered $R$-module over a filtered ring $R$ with filtration $\left\{M_{n}\right\}_{n \geq 0}$ and $\left\{R_{n}\right\}_{n \geq 0}$ respectively. Let $g r_{n}(M)=M_{n} / M_{n+1}$, and $\operatorname{gr}(M)=\oplus_{n \geq 0} g r_{n}(M)$. Then $\operatorname{gr}(M)$ has a natural $\operatorname{gr}(R)$-module structure given by $a+R_{n+1}\left(x+M_{m+1}\right)=a x+M_{m+n+1}$, where $a \in R_{n}, x \in M_{m}$.

\section{Filtered Ring Derived from Discrete Valuation Ring and Its Properties}

In this section we proved that, if $R$ is a discrete valuation ring, then $R$ is a filtered ring. And we prove some properties for $R$.

Let $K$ be a field which $R$ be a domain and a discrete valuation ring (DVR) for $K$. The map $v: K^{*} \rightarrow Z^{+}$is valuation of $R$.

Lemma 3.1 By above definition, the set $R_{n}=\{\alpha \in K \mid v(\alpha) \geq n, n \in Z\}$ is an ideal of $R$.

Proof. (see [3])

Theorem 3.1 If $R$ is a discrete valuation ring with valuation $v: K^{*} \rightarrow Z^{+}$. Then $R$ is a filtered ring with filtration defined by

$$
R_{n}=\{\alpha \in K \mid v(\alpha) \geq n, n \in Z\}
$$

where $R_{0}=R$.

Proof. By definition of valuation ring, it is obvious that $R_{0}=R$. For the second condition for filtration ring we have $\forall \alpha \in R_{n+1} \Rightarrow v(\alpha) \geq n+1>n \Rightarrow v(\alpha)>n \Rightarrow \alpha \in R_{n}$, So we have $R_{n+1} \subseteq R_{n}$.

For the third condition, we have for every $R_{n}$ and $R_{m}$ without losing generality. Since $R_{n}$ and $R_{m}$ are ideals of $R$ so

$$
R_{n} R_{m}=\left\{\sum a_{i} b_{i} \mid a_{i} \in R_{n}-b_{i} \in R_{m}\right\}
$$

is an ideal of $R$.

Now let $c \in R_{n} R_{m}$ then $c=\sum_{i \in I} a_{i} b_{i}$ for $a_{i} \in R_{n}$ and $b_{i} \in R_{m}$.

Thus

$$
v(c)=v\left(\sum_{i \in I} a_{i} b_{i}\right)=\min \left\{v\left(a_{i} b_{i}\right)\right\}_{i \in I}=\min \left\{v\left(a_{i}\right)+v\left(b_{i}\right)\right\}_{i \in I} \geq\left(\min \left\{v\left(a_{i}\right)\right\}_{i \in I}\right)+\left(\min \left\{v\left(b_{i}\right)\right\}_{i \in I}\right) \geq n+m,
$$

Consequently we have $v(c) \geq n+m \Rightarrow c \in R_{n+m}$ hence $R_{n} R_{m} \subseteq R_{n+m}$. Therefore $R$ is a filtered ring.

Proposition 3.1 Let $R$ be a local domain. If every non-zero fractionary ideal of $R$ invertible, then $R$ is filtered ring.

Proof. By proposition $2.1 R$ is DVR then by theorem $3.1 R$ is filtered ring.

Proposition 3.2 Let $R$ be a Noetherian local domain with unique maximal ideal $m \neq 0$ and $K$ the quotient field of $R$. Then $R$ is filtered ring if one of following conditions is held

i) $R$ is a principal ideal domain;

ii) $m$ is principal;

iii) $R$ is integrally closed and every non-zero prime ideal of $R$ is maximal.

Proof. It follows from theorem (3.1) and theorem (2.1).

Definition 3.1 Let $R$ be a ring, and let $(S,+)$ be a totally ordered cancellative semigroup having identity 0 . A function $f: R \rightarrow S \bigcup\{\infty\}$ is a filtration if $f(1)=0, f(0)=\infty$ and for all $x, y \in R$, 
i) $f(x+y) \geq \min \{f(x), f(y)\}$, and

ii) $f(x y) \geq f(x)+f(y)$, then $f$ is called a filtration.

For this filtration we have

1) $\left\{A_{g}: g \in S^{+}\right\}$the set of ideals;

2) $A_{+}=\{x \in R: f(x)>0\}$;

3) $(f)^{g}=\left\{x \in R: \exists n>0\right.$ such that $\left.g \leq f\left(x^{n}\right)\right\}$;

4) $(f)_{g}=\left\{x \in R: \exists n>0\right.$ such that $\left.g=f\left(x^{n}\right)\right\}$.

Lemma 3.2 Let $f: R \rightarrow S \cup\{\infty\}$ be a filtration and let $g, h \in S$. Then:

i) $(f)_{0}=\sqrt{A_{+}}$;

ii) $(f)^{\infty}=0$;

iii) $(f)_{g} \subseteq(f)^{g}$;

iv) if $g \leq h$, then $(f)_{h} \subseteq(f)_{g}$ and $(f)^{h} \subseteq(f)^{g}$.

Proof. See lemma 3.3 of [7].

Proposition 3.3 If $R$ be a discrete valuation ring, then there exists a totally ordered cancellative semigroup $S$, and $f: R \rightarrow S \cup\{\infty\}$ such that:

i) $(f)_{0}=\sqrt{A_{+}}=\sqrt{m}$;

ii) $(f)^{\infty}=0$;

iii) $(f)_{g} \subseteq(f)^{g}$;

iv) if $g \leq h$, then $(f)_{h} \subseteq(f)_{g}$, and $(f)^{h} \subseteq(f)^{g}$.

Proof. By theorem 3.1 there exists a filtration for $R$, then by lemma 2.1 we have the all above conditions.

Proposition 3.4 Let $R$ be a filtered ring, $M, N$ filtered $R$-modules, and $f: M \rightarrow N$ homomorphism of filtered $R$-modules. If the induced map $\operatorname{gr}(f): \operatorname{gr}(M) \rightarrow \operatorname{gr}(N)$ is injective, then $f$ is injective provided $\bigcap_{n=0}^{\infty} M_{n}=(0)$. (see [3])

Corollary 3.1 Let $R$ be a valuation ring, $M, N$ filtered $R$-modules, and $f: M \rightarrow N$ homomorphism of filtered $R$-modules. If the induced map $\operatorname{gr}(f): \operatorname{gr}(M) \rightarrow \operatorname{gr}(N)$ is injective, then $f$ is injective provided $\bigcap_{n=0}^{\infty} M_{n}=(0)$.

Proposition 3.5 If $R$ is a discrete valuation ring with valuation $v: K^{*} \rightarrow Z^{+}$, Then $R$ is a strongly filtered ring with filtration defined by

$$
R_{n}=\{\alpha \in K \mid v(\alpha) \geq n, n \in Z\}
$$

where $R_{0}=R$.

Proof. By theorem $3.1 R$ is a filtered ring. Now we show $R_{n} R_{m}=R n+m$ for all $\alpha \in R_{n+m}$. Since $n+m \geq n, m$ so

$$
R_{m+n} \subseteq R_{n} \text { and } R_{n+m} \subseteq R_{m} .
$$

Consequently $\alpha \in R_{n}$, and $\alpha \in R_{m}$. Therefore $R_{n+m} \subseteq R_{n} R_{m}$.

Proposition 3.6 Let $R$ be a discrete valuation ring, and $f: R \rightarrow S \cup\{\infty\}$. If $x \in R$ and $f(x)>0$, then $(f)^{f(x)}$ is smallest prime ideal in $\operatorname{Spec}_{f}(R)$ which contains $x$, and $(f)_{f(x)}$ is largest prime ideal in $\operatorname{Spec}_{f}(R)$ which does not contains $x$. 
Proof. By proposition 3.5 $R$ is strongly filtered ring, then by proposition 4.2. of [7]-[9] we have If $x \in R$ and $f(x)>0$, then $(f)^{f(x)}$ is smallest prime ideal in $\operatorname{Spec}_{f}(R)$ such that contains $x$, and $(f)_{f(x)}$ is the largest prime ideal in $\operatorname{Spec}_{f}(R)$ such that does not contains $x$.

Remark 3.1 Given a strong filtration $f$ on a ring $R$, we say that a prime $P$ in $\operatorname{Spec}_{f}(R)$ is branched in $\operatorname{Spec}_{f}(R)$, if $P$ cannot be written as union of prime ideals in $\operatorname{Spec}_{f}(R)$ such that properly contained in $P$.

Corollary 3.2 Let $R$ be a discrete valuation ring and $f: R \rightarrow S \cup\{\infty\}$. Then a prime ideal $P$ in $\operatorname{Spec}_{f}(R)$ is branched in $\operatorname{Spec}_{f}(R)$ if and only if $P=(f)^{g}$ for some $g \in S^{+}$.

Proof. By proposition $3.5 R$ is strongly filtered ring, then by proposition 4.5. of [7] a prime ideal $P$ in $\operatorname{Spec}_{f}(R)$ is branched in $\operatorname{Spec}_{f}(R)$, if and only if, $P=(f)^{g}$ for some $g \in S^{+}$.

\section{References}

[1] Atiyah, M.F. and Macdonald, L.G. (1969) Introduction to Commutative Algebra. Addison-Wesley Publishing Company.

[2] Bourbaki, N. (1972) Commutative Algebra. Originally Published as Elements de Mathematique, Algebra Commutative 1964, 1965, 1968, 1969 by Hermann, Paris.

[3] Gopalakrishnan, N.S. (1983) Commutative Algebra. Oxonian Press, PVT, LTD, New Delhi.

[4] Puninskia, G., Puninskayab, V. and Toffalorib, C. (2007) Decidability of the Theory of Modules over Commutative Valuation Domains. Annals of Pure and Applied Logic, 145, 258-275. http://dx.doi.org/10.1016/j.apal.2006.09.002

[5] Cohen, F.R. and Heap, A. (2011) Alexandra Pettet on the Andreadakis Johnson Filtration of the Automorphism Group of a Free Group. Journal of Algebra, 329, 72-91. http://dx.doi.org/10.1016/j.jalgebra.2010.07.011

[6] Levy, R., Loustauna, P. and Shapiro, J. (1991) The Prime Spectrum of an Infinite Product of Copies of Z. Fundamenta Mathematicae, 138, 155-164.

[7] Nishida, K. (2005) On the Depth of the Associated Graded Ring of a Filtration. Journal of Algebra, 285, 182-195. http://dx.doi.org/10.1016/j.jalgebra.2004.10.026

[8] Olberding, B., Saydam, S. and SHapiro, J. (2005) Complitions, Valuations and Ultrapowers of Noetherian Domain. Journal of Pure and Applied Algebra, 197, 213-237. http://dx.doi.org/10.1016/j.jpaa.2004.09.002

[9] Rush, D.E. (2007) Rees Valuations and Asymptotic Primes of Rational Powers in Noetherian Rings and Lattices. Journal of Algebra, 308, 295-320. http://dx.doi.org/10.1016/j.jalgebra.2006.08.014 